\title{
A Chan Dietary Intervention Enhances Executive Functions and Anterior Cingulate Activity in Autism Spectrum Disorders: A Randomized Controlled Trial
}

\author{
Agnes S. Chan, ${ }^{1,2,3}$ Sophia L. Sze, ${ }^{1,2}$ Yvonne M. Y. Han, ${ }^{1,4}$ and Mei-chun Cheung \\ ${ }^{1}$ Neuropsychology Laboratory, Department of Psychology, The Chinese University of Hong Kong, Shatin, NT, Hong Kong \\ ${ }^{2}$ Integrative Neuropsychological Rehabilitation Center, The Chinese University of Hong Kong, Shatin, NT, Hong Kong \\ ${ }^{3}$ Henan Songshan Research Institute for Chanwuyi, Henan 452470, China \\ ${ }^{4}$ Department of Special Education and Counselling, The Hong Kong Institute of Education, Tai Po, Hong Kong \\ ${ }^{5}$ Institute of Textiles and Clothing, The Hong Kong Polytechnic University, Kowloon, Hong Kong \\ Correspondence should be addressed to Agnes S. Chan, aschan@psy.cuhk.edu.hk
}

Received 15 December 2011; Accepted 28 February 2012

Academic Editor: Lixing Lao

Copyright () 2012 Agnes S. Chan et al. This is an open access article distributed under the Creative Commons Attribution License, which permits unrestricted use, distribution, and reproduction in any medium, provided the original work is properly cited.

\begin{abstract}
Executive dysfunctions have been found to be related to repetitive/disinhibited behaviors and social deficits in autism spectrum disorders (ASDs). This study aims to investigate the potential effect of a Shaolin-medicine-based dietary modification on improving executive functions and behavioral symptoms of ASD and exploring the possible underlying neurophysiological mechanisms. Twenty-four children with ASD were randomly assigned into the experimental (receiving dietary modification for one month) and the control (no modification) groups. Each child was assessed on his/her executive functions, behavioral problems based on parental ratings, and event-related electroencephalography (EEG) activity during a response-monitoring task before and after the one month. The experimental group demonstrated significantly improved mental flexibility and inhibitory control after the diet modification, which continued to have a large effect size within the low-functioning subgroup. Such improvements coincided with positive evaluations by their parents on social communication abilities and flexible inhibitory control of daily behaviors and significantly enhanced event-related EEG activity at the rostral and subgenual anterior cingulate cortex. In contrast, the control group did not show any significant improvements. These positive outcomes of a one-month dietary modification on children with ASD have implicated its potential clinical applicability for patients with executive function deficits.
\end{abstract}

\section{Introduction}

Executive dysfunction is a typical cognitive deficit associated with autism spectrum disorders (ASDs) which has been studied for over two decades. Executive functions refer to higher-order cognitive processes, such as working memory, attention, planning, response inhibition, mental flexibility, and self-monitoring. Well-balanced coordination among these various functions is crucial for the efficient execution of goal-directed behaviors. Although there is still debate on whether executive dysfunction really exists in ASD, it has been generally accepted in several recent reviews that deficits in planning, response inhibition, and mental flexibility are three relatively more common executive dysfunctions found in individuals with ASD [1]. Planning ability requires constant monitoring, evaluating, and updating of one's actions to formulate efficient strategies in problem solving. Impairment in planning and strategy formation are relatively prominent in children with ASD at younger ages $(<11$ years) [2] or with mental retardation [3]. Mental inflexibility is more commonly found in ASD individuals with lower intellectual functioning and verbal deficits when they are performing tasks that require flexible shifting of thought and action in response to situational changes [4]. In terms of response inhibition, that is, to execute inhibitory control of irrelevant or interfering information or impulses, individuals with ASD are more likely to demonstrate deficits when the tasks require inhibition of prepotent responses or when the inhibitory process entails more efforts that require additional working memory load [5]. The common ground for these 
three executive functions is a high demand on effective selection and initiation of context-appropriate responses and inhibition of inappropriate/irrelevant responses.

It has been suggested that the executive dysfunctions that involve inefficient and inappropriate selection, initiation, and suppression of thoughts and behaviors account for typical autistic features, such as the strong need for sameness, repetitive behaviors, restricted interests, uncontrollable behavioral and emotional reactions, and impaired social communication and interaction abilities. Some empirical studies have revealed a possible linkage between executive dysfunctions and autistic symptomatology [6-11]. For instance, Kenworthy and colleagues [6] reported significant relationships between both laboratory tasks testing executive functions and behavioral symptoms of ASD. Specifically, the performance of an inhibitory control test was negatively correlated with the severity level of restricted and repetitive behaviors in autism as measured by the Autism Diagnostic Interview and the Autism Diagnostic Observation Schedule. Some other studies [7-9] have also found a significant association between cognitive flexibility or set-shifting and repetitive behaviors in both children and adults with ASD. Another study further reported that perseverative responses in executive functioning tests on young autistic children were correlated with deficits in their social communication skills [10]. Berger et al. [11] found that performance in a cognitive flexibility task was a better predictor of social understanding and competence in autism.

Given that effective pharmacological intervention is not yet available for enhancing the executive functions of ASD, the majority of interventions that target the executive control of emotions and behaviors in autism are primarily behavioral or educational based [12, 13]. Although some behavioral interventions are found to be effective in eliciting context-appropriate behaviors and reducing problematic behaviors or emotional outbursts, the programs tend to be very intensive and time consuming $[14,15]$. For instance, applied behavioral analysis is the most extensively researched educational-behavioral intervention and has been reported to show promising treatment effects on reducing various autistic symptoms. Yet, the most favorable outcomes are suggested to occur when the training programs are started before age 5 and intensively implemented for at least 20 hours per week for two or more years [15]. In addition, children with limited mental abilities who are not able to follow the rules and requirements of the training or have more severe autistic symptoms tend to show less positive outcomes from the many behavioral interventions [16]. Given the limitation of conventional intervention, some researchers have been studying various less conventional and exploratory methods, such as dietary interventions $[17,18]$, nutritional supplements $[19,20]$, music therapy [21-23], massage [24-26], and acupuncture [27, 28]. Although some preliminary data have revealed positive outcomes of these novel interventions, the treatment efficacy remains largely inconclusive [29]. The purpose of the present study is to explore a traditional Chinese concept of healing that has been practiced within the Shaolin temple for over a thousand years and examine its effect on reducing some of the executive dysfunctions in ASD.

The Shaolin temple, although a Buddhism monastery, has been renowned for its martial arts and medicine. The concept of "food as medicine" has been well applied as clinical intervention for promoting mental and physical health within the Shaolin medical approach (Chanyi). It is believed that inappropriate intake of certain types of food may have harmful effects on physical health, mental state, and cognitive functions of human beings. For instance, the Bencao Gangmu written by Li Shizhen during the Ming Dynasty clearly stated that the long-term ingestion of garlic may cause forgetfulness, and excessive intake of ginger can be harmful to the mind and intelligence. The Chanyi proposed that excessive intake of hot and spicy foods (including all meats, seafood, eggs, ginger, garlic, spring onions, Chinese chives, and chili food) with high-fat and high-energy content will generate excessive heat inside the body and cause blood and $Q i$ stagnation, which in turn, result in both physical and mental illnesses.

The Chanyi believed that the body can heal itself. In order to facilitate the self-healing process, it recommends the adoption of a natural and balanced vegetarian diet and reduction of the intake of the mentioned hot and spicy foods. The Chanyi (also termed as Dejian mind-body intervention) [30] has been examined recently in empirical studies and revealed positive results on improving physical health, mood and cognitive functions in community-dwelling adults [31], children with autistic/Asperger's disorder [32, 33], and individuals with brain damage [34] and depression [35, 36]. While previous studies have examined the effects of the holistic approach of Chanyi including psychoeducation, mind-body exercises, and diet modification, the present study aims to examine the effect of a single component within the model, that is, diet modification, as a possible intervention for children with ASD. Given the encouraging findings in previous studies, it is anticipated that a change in diet will reduce some of the executive function deficits (as measured by standardized neuropsychological tests) and related behavioral and social problems (as measured by parental reports on daily behaviors and social communication abilities) in children with ASD in an experimental group, as compared with their counterparts, the control group, who did not change their diet.

In addition, the present study will attempt to explore the possible neural mechanisms that underlie the potential treatment effects of diet modification by examining the pre-post alteration in neuroelectrophysiological responses of ASD children while performing a Go/No-go task. The Go/No-go task is selected because it is a common cognitive test that measures the executive control of response selection, execution, and inhibition, and some previous studies have found deficient performance in this task among ASD individuals [37, 38]. An extensive neural network that covers the prefrontal cortex, anterior cingulate cortex (ACC), and parietal lobes has been found to be activated during the Go/No-go task $[38,39]$. Specifically, the ACC plays a major role in attentional control, error detection, response inhibition, and conflict monitoring [40-42]. Given that our 
TABLE 1: Baseline demographic and clinical characteristics of participants in the control and experimental groups.

\begin{tabular}{|c|c|c|c|c|}
\hline Characteristics & $\begin{array}{l}\text { Control group } \\
\quad(n=12)\end{array}$ & $\begin{array}{l}\text { Experimental group } \\
\qquad(n=12)\end{array}$ & $t / \chi^{2}$ & $P$ value \\
\hline Age (mean $\pm S D)$, years & $12.33 \pm 2.38$ & $11.06 \pm 2.43$ & -1.30 & 0.21 \\
\hline Gender, male (\%) & 83.3 & 83.3 & 0.00 & 1.00 \\
\hline $\mathrm{IQ}($ mean $\pm \mathrm{SD})$ & $60.92 \pm 20.83$ & $73.50 \pm 33.20$ & 1.11 & 0.28 \\
\hline Diagnosis & & & 0.17 & 0.68 \\
\hline Autistic disorder (\%) & 50 & 58.3 & & \\
\hline PDD-NOS (\%) & 50 & 41.7 & & \\
\hline \multicolumn{5}{|l|}{ Severity of disorder $($ mean $\pm \mathrm{SD})$} \\
\hline ADI-R social interaction & $21.50 \pm 5.42$ & $21.00 \pm 8.41$ & -0.17 & 0.86 \\
\hline ADI-R communication & $14.08 \pm 6.16$ & $13.42 \pm 3.99$ & -0.32 & 0.76 \\
\hline ADI-R stereotyped behavior & $4.75 \pm 2.77$ & $3.42 \pm 3.34$ & -1.06 & 0.30 \\
\hline ADI-R abnormal < 36 months & $3.50 \pm 1.00$ & $3.92 \pm 1.44$ & 0.82 & 0.42 \\
\hline
\end{tabular}

ADI-R: Autism Diagnostic Interview-Revised; IQ: intelligence quotient as assessed by the Chinese version of Wechsler Intelligence Scale for Children-Third Edition or the Stanford-Binet Intelligence Scale-Fourth Edition; PDD-NOS: Pervasive Developmental Disorders, not otherwise specified.

recent study has revealed specific significant suppression in event-related theta activity of the ACC during a Go/No-go task in children with ASD [43], together with other repeated empirical evidence for hypoactive ACC in ASD [44-46], it is thus suggested that suppressed ACC activity may be a possible underlying factor for the impaired executive control of behaviors in the ASD population. Therefore, it is anticipated that children who have improved executive functions after dietary modification will demonstrate increased ACC activity during the Go/No-go task, whereas those without intervention will not show this increase.

\section{Methods}

2.1. Study Design. The study was designed as a randomized controlled trial. The recruited children with ASD were randomly and equally assigned into the experimental group (who were required to follow the Chanyi approach in diet modification) or the control group (who were reminded to maintain their regular usual diet). Measurements on various executive functions, behavioral problems, and event-related neurophysiological activities were obtained before and after the one month of changed/unchanged diet regime.

2.2. Participants. Twenty-four children with ASD between the ages of 7 to 17 years old voluntarily participated in the study with written consent from their parents. The children were recruited from the database at the Neuropsychology Laboratory of the Chinese University of Hong Kong. All of the children received formal diagnosis of autistic disorder or pervasive developmental disorders, not otherwise specified by a clinical psychologist through a standard clinical interview with their parents based on the DSM-IV-TR criteria [47]. The degree of severity in autistic symptoms, including those that are related to social interaction, communication, and repetitive/stereotyped behaviors, was assessed by a clinical psychologist with the Autism Diagnostic InterviewRevised (ADI-R) [48]. The interview covers detailed questions about the early development and current functioning of the child, with higher scores indicating more severe autistic symptoms. Children with other neurodevelopmental, psychiatric, or neurological comorbidities or prescribed psychiatric medication were excluded from the study.

The 24 children were randomly and equally assigned into the experimental (with diet modification) or the control group (without diet modification). Table 1 presents the demographic and clinical characteristics of the two groups. Children in both groups were matched on age $[t(22)=$ $-1.296, P=0.21]$, gender $\left[\chi^{2}(1)=0.00, P=1.00\right]$, and severity level of the autistic symptoms as measured by the four ADI-R subscales, $t$ ranges from -1.064 to 0.822 , and $P$ ranges from 0.30 to 0.86 . The two groups also demonstrated comparable levels of general intelligence $[t(22)=1.112$, $P=0.28]$, which were assessed by the research assistant by using the short forms of the Chinese version of the Wechsler Intelligence Scale for Children-Third Edition (WISC-III) [49] or the Stanford-Binet Intelligence Scale-Fourth Edition (SB-FE) [50] for nonverbal children or those who showed a floor effect on the WISC-III. Among the 12 children in each group, 9 in the control group and 8 in the experimental group had limited intelligence with an IQ score at or below 70.

2.3. Procedures. Prior to the baseline assessment, all of the children and their parents were briefed on the procedure of the assessments and informed consents from the parents were obtained. Then, the parents were interviewed by a clinical psychologist on their child's developmental and medical history based on a structured clinical interview. Meanwhile, the children were individually assessed by trained research assistants on their intellectual functioning, executive functions, and scalp electroencephalography (EEG) activities in a quiet room. The clinical psychologist and research assistants who conducted the assessments were blinded to the study design and group assignment. During the EEG recording, each child was required to perform a Go/No-go task while their EEG data were obtained by using a TruScan measuring set through 19 electrodes positioned across the 
scalp according to the International 10-20 System [51]. With electrode impedances maintained at $\leq 10 \mathrm{k} \Omega$, the EEG signals were referenced to linked ears and sampled at 256 samples per second, with a high-frequency limit band pass of $30 \mathrm{~Hz}$, and then fast Fourier transformed. Details of the Go/No-go task will be elaborated in a later section. Artifactfree EEG data were selected based on visual examination for eye movements and muscle artifacts and then captured for subsequent low-resolution electromagnetic tomography (LORETA) analyses.

After the baseline assessments, the parents of the children in the experimental group were guided on a specific diet modification based on the Chanyi approach for one month, whereas parents of the children in the control group were recommended to maintain their usual dietary habits during the study period. After one month, the same assessments on the executive functions and EEG activity were performed on all of the children. Their parents were also requested to rate their child's executive-function-related behaviors (including social communication problems and repetitive/disinhibited behaviors) in daily life before and after the intervention.

2.4. Neuropsychological Assessments. Four major aspects of executive functions, including attention, mental flexibility, response inhibition, and planning, were measured by standardized neuropsychological tests before and after the intervention.

2.4.1. D2 Test of Concentration (D2) [52]. This is a test on attention and inhibitory control that involves 14 lines of the letter "d" or " $\mathrm{p}$ " with a different number of dashes above and/or below the letter. The child was required to cancel as many "d"s with 2 dashes as possible within 20 seconds for each line, while ignoring the nontarget distractors. The concentration performance score which measures the degree of accuracy was used as a measure of attention. The number of commission errors (i.e., incorrect cancellation of distractors) was counted as a measure of disinhibition.

2.4.2. Go/No-Go Task. This is a computerized task to test the ability to attend to and flexibly respond to changing stimuli and inhibit unwanted responses. A total of 192 black balls and 48 red balls (black: red ratio $=4: 1$ ) were randomly displayed, one at a time, for $500 \mathrm{~ms}$ followed by $1000 \mathrm{~ms}$ of blank intervals, in the center of a computer screen. The total testing time was 6 minutes. The child was required to press a key as quickly as possible in response to a black ball (Go stimulus), but to inhibit their response when a red ball (No-go stimulus) appeared. The omission errors at the Go condition were used as a measure of attention, whereas the commission errors during the No-go condition were used as an indicator of disinhibition.

2.4.3. Children's Color Trails Test (CCTT) [53]. The second trial of the CCTT was adopted as a test of mental flexibility. It involves duplicates of each number embedded within pink and yellow circles and requires the child to connect the numbers in ascending order from 1 to 15 while alternating between the two colors as quickly as possible. The completion time was used as a measure of mental flexibility.

2.4.4. The Five-Point Test (FPT) [54]. This figural fluency test was adopted as a test for mental flexibility, as it requires spontaneous generation of novel designs without repetition by connecting five points with straight lines within 5 minutes. A greater number of unique designs generated indicates higher flexibility.

2.4.5. The Tower of California (ToC) Test [55]. The ToC was adopted as a test of planning ability, strategy formulation, and inhibition. It consists of nine items that involve moving discs on three colored vertical pegs to match a target arrangement while adhering to rules. The total achievement score, which is composed of accurate and efficient movements and successful completion of items, was calculated as a measure of planning.

2.5. Parental Evaluation on Behavioral Measures. Given that executive dysfunctions in autism was found to be associated with repetitive/disinhibitory behaviors and social communication abilities, the behavioral changes in ASD children in the present study were examined based on parental evaluation. Their verbal and nonverbal social communication abilities were measured by two subscales from the Autism Treatment Evaluation Checklist (ATEC) [56], namely, communication and sociability, on a 3 -point scale (from " 0 " to "2"), in which a higher score indicates greater problems. The parents were asked to rate a total of 14 items in the communication subscale and 20 items in the sociability subscale before and after the one-month period. The total scores of the two subscales were averaged for a pre-post comparison of each group. For repetitive/disinhibitory behaviors, the parents were asked to rate based on a questionnaire composed of 7 questions that measured the problems of controlling repetitive speech/acts, rigid thoughts/acts, and hyperactive behaviors of their child. They were required to evaluate the degree of change in each behavior problem of their child after one month by using a scale of " -5 " to " +5 ", where " +5 " indicates "largely improved", " -5 " indicates "largely declined," and " 0 " indicates "no change". The average score obtained was used for subsequent comparisons.

2.6. EEG Assessments. The EEG data of each child was first transformed by Excel application before imported into EEGLAB software by using MatLab 7.1 to capture the correct events and epochs. The epoch limit was set as $50 \mathrm{~ms}$ as the start and $900 \mathrm{~ms}$ as the end. Artifacts in epoched data were then pruned by visual inspection and by using the rejection method on the EEG Plot. All incorrect hits were also deselected. The transformed data were exported and then spectrally processed by using fast Fourier transformation (FFT) to compute the power data for the theta band (4$7.5 \mathrm{~Hz}$ ) through the use of NeuroGuide software. Given that the ACC has been reported as one of the generators for theta activities in the human brain [57-59], LORETA $[60,61]$ was adopted to localize the sources of the theta activities in 
response to the "Go" and "No-go" conditions. The sources of the theta activities were expressed as three-dimensional cortical current density according to the Talairach brain atlas.

2.7. Intervention: Specific Diet Modification. Children in the experimental group were recommended to reduce their intake of some foods (including ginger, garlic, green onion, spicy foods, eggs, meat, and fish) which will generate excessive internal heat and adversely affect the temper and cognitive functions. It should be stressed that the children were not required to abstain from these foods, but advised to cut down on their intake according to their own lifestyles and plans. The parents were advised to gradually change their child's diet and required to complete dietary log sheets to verify and monitor their progress weekly. To ensure a wellbalanced nutritional diet, the children in the experimental group were also encouraged to take food from the seven categories every day with one to three kinds from each category. The seven categories are (1) grains (e.g., noodles, brown rice); (2) vegetables (e.g., broccoli, tomatoes); (3) fruits (e.g., grapes, apples); (4) beans (e.g., soy, peas); (5) mushrooms (e.g., black fungus, straw mushrooms); (6) nuts (e.g., walnuts, almonds); (7) roots (e.g., potatoes, yams). The type and amount of food were not specified, as long as they were fresh and seasonal and the children ate until they were $80 \%$ full for each meal. The log record revealed that $33 \%$ of the children completely followed the dietary recommendation, that is, reducing intake of or abstaining from all hot and spicy foods and consuming from all the above seven recommended food categories. Eightynine percent of the children reduced or abstained from consumption of ginger, garlic, green onion, and spicy foods, and $78 \%$ had some meat, fish, and egg throughout the one-month period. The control group received no dietary recommendation and was reminded to maintain the same dietary habits throughout the study.

2.8. Data Analyses. The performance on various neuropsychological measures on executive functions before and after the one month was compared for each group of children by using repeated measures ANOVA and then followed by post hoc paired $t$-tests. The corresponding effect sizes were also computed for comparison between groups. Within- and between-group comparisons on parental rating of changes in the behaviors of their children after one month were also performed using paired and independent sample $t$ tests. To examine the effect of diet modification on neural activity, LORETA voxel-by-voxel paired-sample $t$-tests were performed on EEG data with subjectwise normalization and log transformation for each group of children in comparing the pre-post changes in sources of theta activity at the ACC during the "Go" and "No-go" conditions. Given that specific hypotheses were tested and the number of participants was relatively small, no adjustment to the alpha level was applied to avoid lowering the power of the tests. The effect size was provided to evaluate the extent of the effect of the treatment.

\section{Results}

3.1. Diet Change Improved Some of the Executive Functions in ASD Children. At the baseline, the control and the experimental groups showed comparable levels of functioning in attention, mental flexibility, response inhibition, and planning as measured by the 7 neuropsychological measures, $t$ s range $=-0.91$ to $1.26, P>0.05$, without significant differences between the two groups in any of the measures. Table 2 presents the mean performance of each group on various executive functioning tests before and after the one-month period. The results of the repeated measures ANOVAs showed significant time (pre versus post) by group (experimental versus control) interaction effect on one flexibility measure only (FPT: $F=8.06, P=0.01$ ) and significant main effect of time on an attention measure (D2-concentration performance: $F=5.04, P=0.04$ ) and another flexibility measure (CCTT: $F=9.29, P=0.01$ ). Given that the nonsignificant multivariate results may be possibly due to large within-group variations (as revealed in Table 2 in which the standard deviations in the majority of the measures are approaching or even greater than the group mean), paired-sample $t$-tests were thus performed to explore the trend of pre-post differences in each group. After onemonth modification in diet, the experimental group showed significantly improved performance in the three executive function domains, including mental flexibility (FPT: $t=$ 3.614, $P=0.004$; CCTT: $t=-2.647, P=0.017$ ), response inhibition (D2: $t=-2.771, P=0.016$ ), and planning (ToC: $t=2.072, P=0.039)$. The effect size of these three measures on mental flexibility and inhibitory control with positive treatment effects was large (0.94 to 1.20$)$, and the planning measure had a medium effect size (0.73). The extent of improvement in the performance of the experimental group ranged from $37 \%$ to $62 \%$. In contrast, the control group did not show significant improvement in any of the domains, $t$ s range $=-0.42$ to $1.59, P>0.05$, effect size $=0.03$ to 0.56 , with the extent of improvement ranging from $3 \%$ to $29 \%$. In summary, diet modification based on the Chanyi approach for one month has positive effects on improving mental flexibility, response inhibition, and planning in children with autism.

For the measure of attention ability, both the experimental and control groups demonstrated a trend of improvement in the concentration performance score of the D2 test with a medium effect size (0.69 and 0.54$), t=1.83$ and 1.60 , $P=0.059$ and 0.074 , respectively. The experimental group also showed a reduced number of omission errors in the Go/No-go task (from 38.0 to 31.8) with a small effect size of 0.42 , although this was not significant, $t=-1.33, P=0.109$. However, the control group had more omissions after one month (from 20.2 to 25.0), $t=1.01, P=0.170$, although this was not significant. It seems that diet modification has a smaller extent of positive effects on the attention ability of ASD children.

Given that diet modification has significant positive effects on the four measures of mental flexibility, inhibitory control, and planning, pre-post analyses on these four measures were performed on the low-functioning subgroups 
TABLE 2: Mean performance in executive functioning of the control and experimental groups at before and after one-month period.

\begin{tabular}{|c|c|c|c|c|c|c|c|c|}
\hline & \multicolumn{2}{|c|}{ Control group $(n=12)$} & \multirow{2}{*}{$P$ value } & \multirow{2}{*}{ Effect size } & \multicolumn{2}{|c|}{ Experimental group $(n=12)$} & \multirow{2}{*}{$P$ value } & \multirow{2}{*}{ Effect size } \\
\hline & Before & After & & & Before & After & & \\
\hline \multicolumn{9}{|l|}{ Attention } \\
\hline D2: concentration performance & $\begin{array}{c}78.67 \\
(41.33)\end{array}$ & $\begin{array}{c}98.22 \\
(61.23)\end{array}$ & 0.074 & $0.535^{+}$ & $\begin{array}{c}92.86 \\
(50.47)\end{array}$ & $\begin{array}{l}109.14 \\
(53.00)\end{array}$ & 0.059 & $0.690^{+}$ \\
\hline Go/No-go: omission ${ }^{\#}$ & $\begin{array}{c}20.20 \\
(22.08)\end{array}$ & $\begin{array}{c}25.00 \\
(30.67)\end{array}$ & 0.170 & 0.319 & $\begin{array}{c}38.00 \\
(43.06)\end{array}$ & $\begin{array}{c}31.80 \\
(38.32)\end{array}$ & 0.109 & 0.421 \\
\hline \multicolumn{9}{|l|}{ Mental flexibility } \\
\hline FPT: unique design & $\begin{array}{l}15.67 \\
(9.29)\end{array}$ & $\begin{array}{l}16.22 \\
(8.29)\end{array}$ & 0.320 & 0.137 & $\begin{array}{c}12.56 \\
(11.36)\end{array}$ & $\begin{array}{c}20.33 \\
(15.84)\end{array}$ & $0.004^{* *}$ & $1.204^{++}$ \\
\hline CCTT-T2: time (in seconds) & $\begin{array}{c}94.09 \\
(72.17)\end{array}$ & $\begin{array}{c}67.18 \\
(32.64)\end{array}$ & 0.078 & $0.562^{+}$ & $\begin{array}{l}132.08 \\
(98.00)\end{array}$ & $\begin{array}{c}78.73 \\
(51.44)\end{array}$ & $0.017^{*}$ & $0.936^{++}$ \\
\hline \multicolumn{9}{|l|}{ Response inhibition } \\
\hline D2: commission ${ }^{\#}$ & $\begin{array}{c}22.00 \\
(27.24)\end{array}$ & $\begin{array}{c}21.44 \\
(29.05)\end{array}$ & 0.461 & 0.034 & $\begin{array}{c}23.57 \\
(38.21)\end{array}$ & $\begin{array}{c}14.86 \\
(32.32)\end{array}$ & $0.016^{*}$ & $1.047^{++}$ \\
\hline Go/No-go: commission ${ }^{\#}$ & $\begin{array}{c}13.00 \\
(11.30)\end{array}$ & $\begin{array}{c}11.40 \\
(7.40)\end{array}$ & 0.216 & 0.261 & $\begin{array}{c}13.27 \\
(11.47)\end{array}$ & $\begin{array}{c}15.27 \\
(13.66)\end{array}$ & 0.204 & 0.260 \\
\hline \multicolumn{9}{|l|}{ Planning } \\
\hline ToC: achievement score & $\begin{array}{c}5.70 \\
(2.41)\end{array}$ & $\begin{array}{c}6.20 \\
(3.97)\end{array}$ & 0.344 & 0.131 & $\begin{array}{c}6.25 \\
(3.41)\end{array}$ & $\begin{array}{c}8.88 \\
(5.28)\end{array}$ & $0.039^{*}$ & $0.733^{+}$ \\
\hline
\end{tabular}

FPT: five-point test; D2: D2 Test of Attention; CCTT-T2: time: total completion time in trial 2 of the Children's Color Trails Test; ToC: Tower of California Test. Standard deviations are in parenthesis. ${ }^{\#}$ Lower value indicates better performance; ${ }^{*} P<0.05,{ }^{* *} P<0.01$; ${ }^{+}$medium effect size, ${ }^{++}$large effect size.

TABLE 3: Mean performance in executive functioning of the control and experimental low-functioning subgroups at before and after onemonth period.

\begin{tabular}{|c|c|c|c|c|c|c|c|c|}
\hline & \multicolumn{2}{|c|}{ Control Group $(n=9)$} & \multirow[t]{2}{*}{$P$ value } & \multirow[t]{2}{*}{ Effect size } & \multicolumn{2}{|c|}{ Experimental Group $(n=8)$} & \multirow[t]{2}{*}{$P$ value } & \multirow[t]{2}{*}{ Effect size } \\
\hline & Before & After & & & Before & After & & \\
\hline \multicolumn{9}{|l|}{ Mental Flexibility } \\
\hline FPT: unique design & $\begin{array}{l}11.00 \\
(6.99)\end{array}$ & $\begin{array}{l}11.67 \\
(4.80)\end{array}$ & 0.353 & 0.163 & $\begin{array}{c}4.60 \\
(4.34)\end{array}$ & $\begin{array}{c}8.80 \\
(6.18)\end{array}$ & 0.084 & $0.752^{+}$ \\
\hline CCTT-T2: time ${ }^{\#}$ & $\begin{array}{l}112.34 \\
(89.16)\end{array}$ & $\begin{array}{c}71.88 \\
(41.88)\end{array}$ & 0.098 & $0.695^{+}$ & $\begin{array}{l}184.25 \\
(98.48)\end{array}$ & $\begin{array}{l}110.27 \\
(56.28)\end{array}$ & $0.032^{*}$ & $1.448^{++}$ \\
\hline \multicolumn{9}{|l|}{ Response Inhibition } \\
\hline D2: commission ${ }^{\#}$ & $\begin{array}{c}26.00 \\
(33.11)\end{array}$ & $\begin{array}{c}23.67 \\
(34.06)\end{array}$ & 0.384 & 0.128 & $\begin{array}{c}43.33 \\
(56.01)\end{array}$ & $\begin{array}{c}31.33 \\
(49.07)\end{array}$ & $0.049^{*}$ & $1.710^{++}$ \\
\hline \multicolumn{9}{|l|}{ Planning } \\
\hline ToC: achievement score & $\begin{array}{c}5.29 \\
(2.43)\end{array}$ & $\begin{array}{c}4.86 \\
(3.34)\end{array}$ & 0.309 & 0.199 & $\begin{array}{c}3.75 \\
(2.36)\end{array}$ & $\begin{array}{c}5.75 \\
(4.27)\end{array}$ & 0.228 & 0.426 \\
\hline
\end{tabular}

FPT: five-point test; D2: D2 Test of Attention; CCTT-T2: time: total completion time in trial 2 of the Children's Color Trails Test; ToC: Tower of California Test. Standard deviations are in parenthesis. ${ }^{\#}$ Lower value indicates better performance; ${ }^{*} P<0.05 ;{ }^{+}$medium effect size, ${ }^{++}$large effect size.

(i.e., IQ $\leq 70$ ) to explore if a similar treatment effect is replicable on them. There were 8 and 9 low-functioning children in the experimental and control groups, respectively. The results of paired $t$-tests showed that a similar extent of improvement in all three measures of flexibility and inhibition was replicable in the experimental lowfunctioning subgroup (with a medium to large effect size), and the control subgroup consistently showed no significant improvement in any of the measures (Table 3 ). Even with the small sample size, the performance of the two measures on mental flexibility and response inhibition remained statistically significant with a large effect size (CCTT-T2: time: $t=-2.89, P=0.032$; D2: commission: $t=-2.97, P=$
0.049). For the performance change in the ToC (the planning test) of the experimental group, the effect size was reduced from previously medium (0.73) in the analysis for the entire group, to small $(0.43)$ in the subgroup analysis. This finding has provided preliminary support for the positive effect of diet change in mental flexibility and response inhibition of low-functioning ASD children.

3.2. Parental Evaluation on Daily Behavioral Control and Social Communication after Diet Modification. In terms of the average score of the pre-post comparison for the ATEC communication and sociability subscales, the evaluations of parents with children in the experimental group indicated 


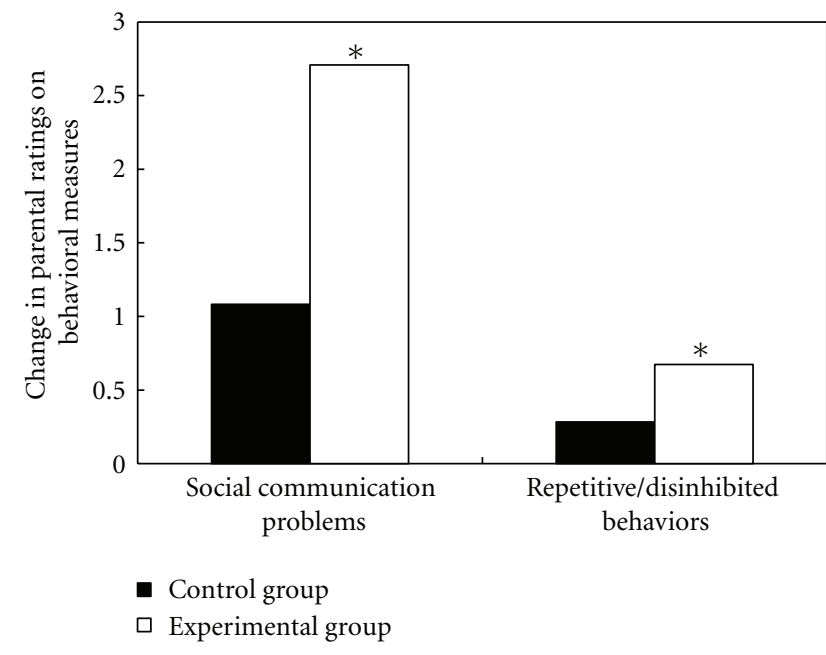

Figure 1: Changes in parental ratings on social communication problems and repetitive/disinhibited behaviors of their children after a one-month period. A positive value indicates improvement in the corresponding problem. ${ }^{*} P<0.05$ (paired-sample $t$-test or one sample $t$-test).

that they significantly demonstrated fewer social communication problems after the diet change for one month, $t(11)$ $=3.898, P=0.001$, effect size $=1.12$. Such significant improvement was not reported by parents of the control group children, $t(11)=1.324, P=0.106$, effect size $=0.38$ (Figure 1). The experimental group demonstrated a $21 \%$ reduction in problematic social behaviors, which was twice that of the control group (11\%).

In addition, parents of the children in the experimental group reported a significantly positive change (mean rating $=0.67$ ) in the executive control of repetitive, inflexible, and hyperactive behaviors of their children after intervention, $t(11)=1.871, P=0.044$, effect size $=0.54$, whereas children in the control group were not reported to have any significant behavioral changes (mean rating $=0.28$ ) by their parents, $t(11)=1.011, P=0.167$, effect size $=0.29$ (Figure 1). While $58 \%$ of the children in the experimental group received improved ratings, $25 \%$ of their counterparts in the control group received improved ratings, but $17 \%$ were evaluated to have experienced deterioration. This suggests that diet modification was considered by the parents of ASD children to have some positive effects on improving their child's daily behavioral control and social communication abilities.

3.3. Diet Change Enhanced Theta Activity in Anterior Cingulate Cortex of ASD Children. Given that the effect of diet modification seems to be more pronounced in the flexible inhibitory control of responses in ASD, further pre-post comparisons on EEG activities during the Go/No-go task (a common test of inhibitory control) were performed. LORETA voxel-by-voxel paired $t$ statistics were performed for each group of children during the Go and No-go conditions to examine the effect of diet modification in enhancing activity level in the ACC. The ACC was selected as the region of interest because our previous study has revealed hypoactive ACC patterns in ASD children in both Go and No-go conditions, as compared to their normal counterparts [43] (circled regions in Figure 2(a)).

At the baseline, the voxel-by-voxel independent $t$ statistics showed that both the control and experimental groups have comparable activity levels in the ACC at both the Go and No-go conditions, $P=0.538$ and 0.609 , respectively. After one month of change in diet, the experimental group showed significantly elevated activity specifically at the rostral ACC regions (Brodmann areas 24 and 32) during the Go condition, maximal $t=1.89, P<0.05$ (circled region in the upper image of Figure 2(b)). This specific region with increased activity after the diet change was the region that showed hypoactivity in the ASD children of our previous study (circled region in the upper image of Figure 2(a)). In contrast, the control group did not show significant activity change in the rostral ACC, maximal $t=1.25, P>$ 0.05 . During the No-go condition, the experimental group showed significantly increased activity in the subgenual ACC region (Brodmann area 25) and subcallosal gyrus after onemonth intervention (circled region in the lower image of Figure 2(b)), maximal $t=2.04, P<0.05$, which were also the regions that showed hypoactivity in ASD children in our previous study (circled region in the lower image of Figure 2(a)). For the control group, there was no significant change in ACC activity after one month, maximal $t=0.24$, $P>0.05$.

\section{Discussion}

Typical symptoms of ASD, including repetitive mannerisms, impulsive acts, emotional outbursts, restricted interests, inflexible adherence to specific routines, and social communication deficits, were found to be correlated with executive dysfunctions in response selection, alteration, and inhibition. Intervention that can reduce the executive dysfunctions of children with ASD may reduce their behavioral problems in daily life. The present findings showed that a specific diet modification based on the Chinese Chan medical approach had some positive effects on improving executive functions and typical behavioral symptoms of children with ASD. These results are encouraging since commonly used behavioral interventions for ASD children are very time consuming and not cost-effective, while the present diet modification is less time consuming and more economical.

The present finding may suggest an alternative or complementary intervention for the executive control of behaviors among ASD children. It should be noted that the positive effect of diet modification was also applicable to the low-functioning children with IQ at or below 70 in this experiment. Specifically, there were 8 low-functioning children in the experimental group and 9 in the control group. Those in the experimental group showed improvement in tests of mental flexibility and inhibitory control with a large effect size (although some pre-post differences did not reach significance due to the small sample size). However, the lowfunctioning children in the control group did not show such improvement. Given that behavioral training is particularly difficult and time and labor intensive when applied on 
ASD versus NC

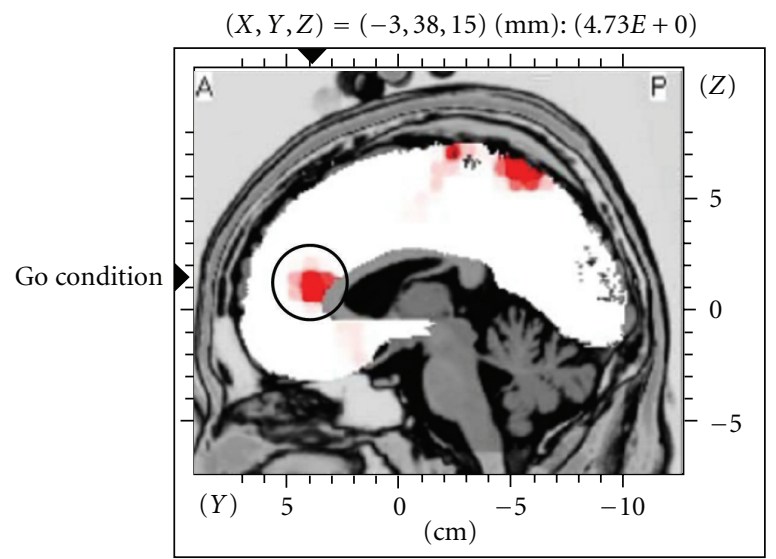

(a)

Control group
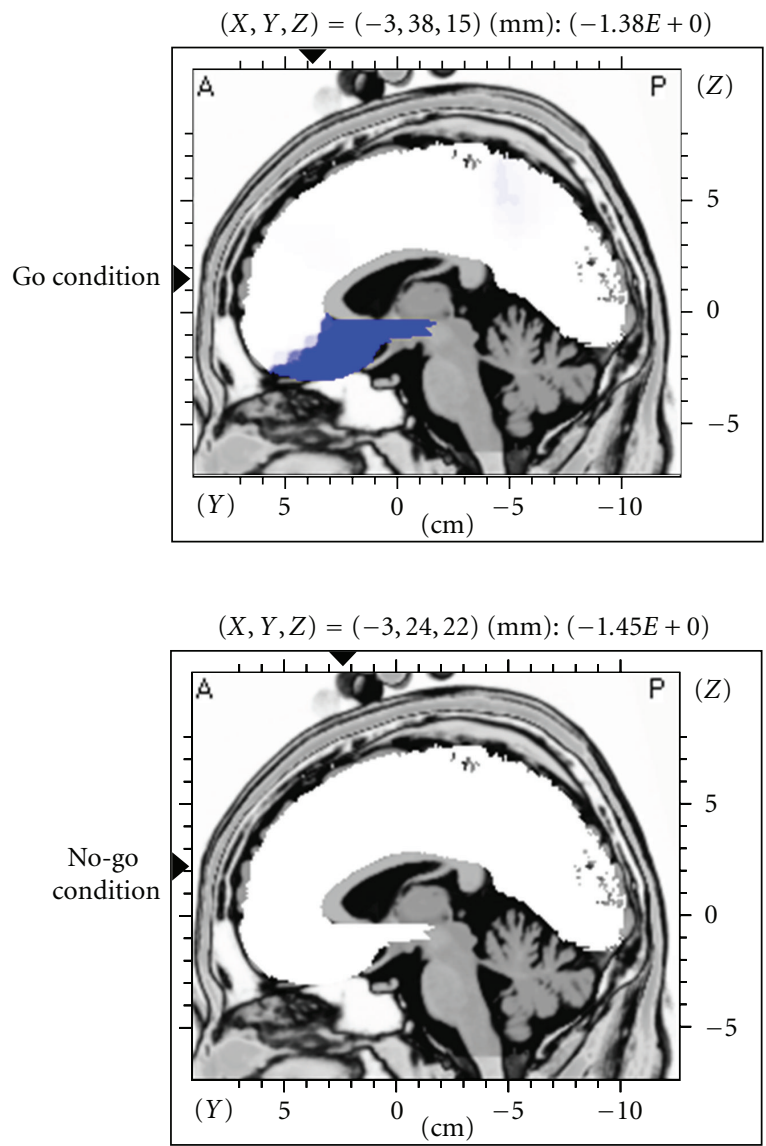

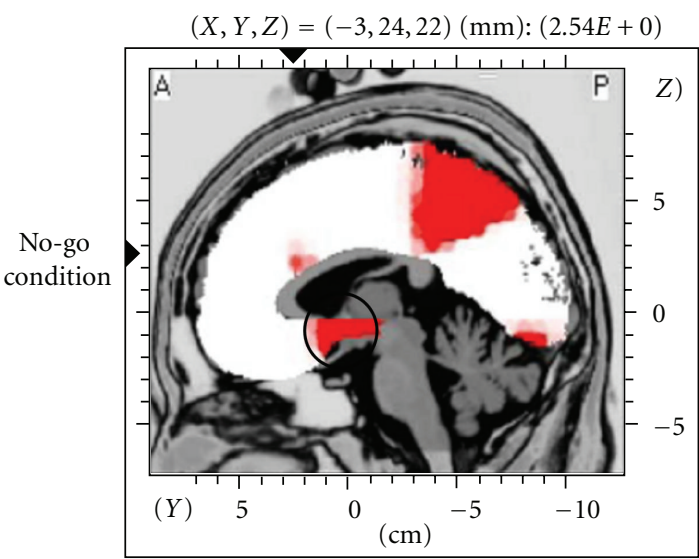

Experimental group

$(X, Y, Z)=(-3,38,15)(\mathrm{mm}):(-1.89 E+0)$

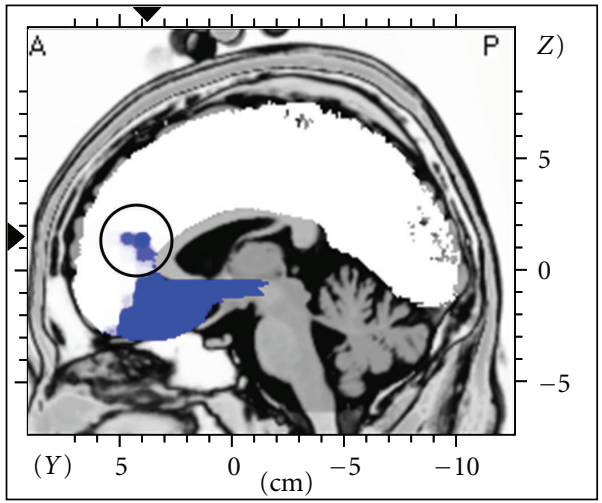

$(X, Y, Z)=(-3,24,22)(\mathrm{mm}):(1.74 E+0)$

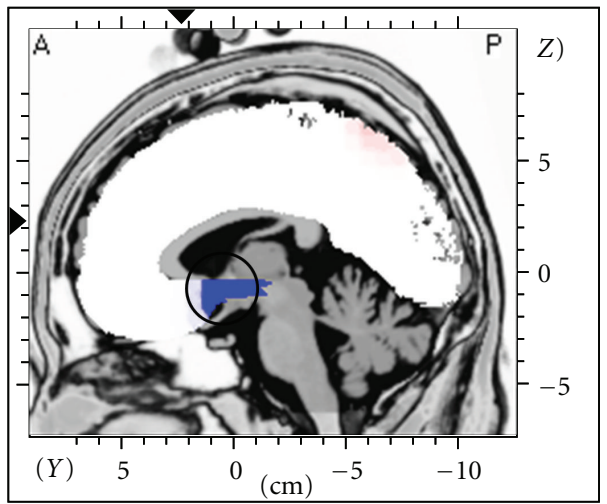

(b)

FIgURE 2: (a) Graphical representation of LORETA independent $t$-statistics results adopted from the research article by Chan et al. [43] published in the Research in Autism Spectrum Disorders. The location of the voxel is defined by the Talairach coordinates $(X, Y, Z)$, and the regions colored in red represent significant hypoactivity in the anterior cingulate cortex (ACC, in the circle) of children with autism spectrum disorders (ASDs) as compared to their normal counterparts (NCs) during the Go and No-go conditions. (b) Graphical representation of LORETA paired $t$-statistics results that compares the pre- and post-one-month theta source activity of the control and experimental groups during the Go and No-go conditions. The regions colored in blue indicate significantly elevated ACC activity after one month at $P$ (onetailed) $<0.05$. The ACC regions (in the circle), in which hypoactivity was previously found in children with autism spectrum disorders, were enhanced in activity after diet modification in the experimental group. 
children with limited intelligence, diet modification that can be monitored by parents may therefore be considered as an alternative.

The underlying mechanism that may explain the change in behaviors was evaluated with an electrophysiological method. LORETA localization of theta activity showed that after one month of diet modification, the ASD children demonstrated increased activation of the ACC specific to the rostral and subgenual subdivisions. The control group did not show such improvement. Our previous study found that ASD children demonstrated hypoactivity in the ACC when performing a response-monitoring and inhibitory task (i.e., Go/No-go task) as compared to normally developed children [43]. The present study showed that a one-month diet modification was able to enhance the activity of the ACC while the child was performing the same task. This finding suggests that the behavioral improvement of children with ASD may be associated with increased activity in the ACC; however, how diet can change and improve activity in the neural system warrants further investigation in future studies.

The therapeutic effects of diet modification on brain functions and activity in the present study are consistent with our decades of clinical observations and some empirical findings in past years. For instance, two studies have found significantly enhanced frontal activity and improved attention in community-dwelling adults with depressed mood and patients with clinical depression after receiving the Dejian mind-body intervention with diet modification as one of the treatment components $[31,35,36]$. Another case study on a low-functioning autistic child also demonstrated significantly improved inhibitory control and cognitive flexibility and increased EEG cordance (an index associated with cerebral perfusion) of the whole brain after Dejian mindbody intervention [32]. Thus, the present study has shed some light on the potential application of the Chinese Chanbased diet modification as a complementary intervention for rehabilitation of individuals with ASD and other individuals with emotional and cognitive problems.

The idea of food as medicine has also drawn increasing attention in western scientific research. A number of studies have supported the beneficial effects of a vegetarian or vegan diet in promoting the health of cardiovascular and digestive systems, reducing cancers and degenerative diseases and improving mood [62-64]. Some studies have also revealed a significant linkage between a balanced nutritional diet and level of cognitive functions and cognitive development in early life $[65,66]$. However, many of them are observational studies and there is also counterevidence against the positive dietary effects [67]. Therefore, it remains inconclusive in terms of the actual outcomes and the choice of type of diet.

The present study has shown the positive effects of a onemonth dietary modification on the executive functions and autistic symptoms of children with autism; however, its longterm effect is still unknown which is worth further investigation. In addition, the sample size of the low-functioning subgroup is relatively small; therefore, future studies with larger sample sizes will be helpful to verify the effect of diet modification. Given the preliminary evidence on the effects of diet change in the low-functioning subgroups, it will also be worth investigating if this can benefit patients with severe brain disorders or physical disabilities (e.g., demented or stroke patients) in a well-controlled study. Last but not least, given the increasing research interest in food as medicine to improve health in Western countries, the applicability and effectiveness of this Chinese Chan-based dietary modification to the Caucasian population can also be investigated in future studies.

\section{Conclusion}

This study has provided some evidence for the therapeutic effect of a Chinese Chan-medicine-based diet modification carried out in the time span of one month on improving executive functions, reducing autistic symptoms and enhancing the ACC activity of children with ASD. Such encouraging findings have shed some light on the potential applicability of this specific diet modification for people who suffer from executive function deficits.

\section{Acknowledgments}

This study was partly supported by a donation from Mr. Sau Hung Li to The Chinese University of Hong Kong and partly by the Niche Areas Funding (J-BB6S) from The Hong Kong Polytechnic University. The authors would like to especially thank Venerable Master Dejian of the Sanhuangzhai Monastery for his effort in developing and generosity in sharing the Chinese Chan-medicine-based mind-body intervention. They are also thankful to the parents who have participated in this study and assisted in recruiting participants. Appreciation is also extended to Debbie Yan, Lan He, Man-ying Mo, Beth Chiu, Eric Chan, Queenie Wong, and Connie Leung for their efforts in data collection and data management.

\section{References}

[1] L. Kenworthy, B. E. Yerys, L. G. Anthony, and G. L. Wallace, "Understanding executive control in autism spectrum disorders in the lab and in the real world," Neuropsychology Review, vol. 18, no. 4, pp. 320-338, 2008.

[2] F. Happé, R. Booth, R. Charlton, and C. Hughes, "Executive function deficits in autism spectrum disorders and attention-deficit/hyperactivity disorder: examining profiles across domains and ages," Brain and Cognition, vol. 61, no. 1, pp. 25-39, 2006.

[3] M. Mari, U. Castiello, D. Marks, C. Marraffa, and M. Prior, "The reach-to-grasp movement in children with autism spectrum disorder," Philosophical Transactions of the Royal Society B, vol. 358, no. 1430, pp. 393-403, 2003.

[4] S. Robinson, L. Goddard, B. Dritschel, M. Wisley, and P. Howlin, "Executive functions in children with autism spectrum disorders," Brain and Cognition, vol. 71, no. 3, pp. 362-368, 2009.

[5] E. L. Hill, "Executive dysfunction in autism," Trends in Cognitive Sciences, vol. 8, no. 1, pp. 26-32, 2004.

[6] L. Kenworthy, D. O. Black, B. Harrison, A. Della Rosa, and G. L. Wallace, "Are executive control functions related to autism symptoms in high-functioning children?" Child Neuropsychology, vol. 15, no. 5, pp. 425-440, 2009. 
[7] B. R. Lopez, A. J. Lincoln, S. Ozonoff, and Z. Lai, "Examining the relationship between executive functions and restricted, repetitive symptoms of autistic disorder," Journal of Autism and Developmental Disorders, vol. 35, no. 4, pp. 445-460, 2005.

[8] M. South, S. Ozonoff, and W. M. Mcmahon, "The relationship between executive functioning, central coherence, and repetitive behaviors in the high-functioning autism spectrum," Autism, vol. 11, no. 5, pp. 437-451, 2007.

[9] B. E. Yerys, G. L. Wallace, B. Harrison, M. J. Celano, J. N. Giedd, and L. E. Kenworthy, "Set-shifting in children with autism spectrum disorders: reversal shifting deficits on the intradimensional/extradimensional shift test correlate with repetitive behaviors," Autism, vol. 13, no. 5, pp. 523-538, 2009.

[10] R. E. McEvoy, S. J. Rogers, and B. F. Pennington, "Executive function and social communication deficits in young autistic children," Journal of Child Psychology and Psychiatry and Allied Disciplines, vol. 34, no. 4, pp. 563-578, 1993.

[11] H. J. C. Berger, F. H. T. M. Aerts, K. P. M. Van Spaendonck, A. R. Cools, and J. P. Teunisse, "Central coherence and cognitive shifting in relation to social improvement in highfunctioning young adults with autism," Journal of Clinical and Experimental Neuropsychology, vol. 25, no. 4, pp. 502-511, 2003.

[12] National Research Council, Educating Children with Autism, National Academy Press, Washington, DC, USA, 2001.

[13] R. P. Goin-Kochel, B. J. Myers, and V. H. Mackintosh, "Parental reports on the use of treatments and therapies for children with autism spectrum disorders," Research in Autism Spectrum Disorders, vol. 1, no. 3, pp. 195-209, 2007.

[14] B. Remington, R. P. Hastings, H. Kovshoff et al., "Early intensive behavioral intervention: outcomes for children with autism and their parents after two years," American Journal on Mental Retardation, vol. 112, no. 6, pp. 418-438, 2007.

[15] L. A. Vismara and S. J. Rogers, "Behavioral treatments in autism spectrum disorder: what do we know?" Annual Review of Clinical Psychology, vol. 6, pp. 447-468, 2010.

[16] J. L. Matson and K. R. M. Smith, "Current status of intensive behavioral interventions for young children with autism and pdd-nos," Research in Autism Spectrum Disorders, vol. 2, no. 1, pp. 60-74, 2008.

[17] A. Mulloy, R. Lang, M. O’Reilly, J. Sigafoos, G. Lancioni, and M. Rispoli, "Gluten-free and casein-free diets in the treatment of autism spectrum disorders: a systematic review," Research in Autism Spectrum Disorders, vol. 4, no. 3, pp. 328-339, 2010.

[18] P. Srinivasan, "A review of dietary interventions in autism," Annals of Clinical Psychiatry, vol. 21, no. 4, pp. 237-247, 2009.

[19] J. B. Adams and C. Holloway, "Pilot study of a moderate dose multivitamin/mineral supplement for children with autistic spectrum disorder," Journal of Alternative and Complementary Medicine, vol. 10, no. 6, pp. 1033-1039, 2004.

[20] D. A. Rossignol, "Novel and emerging treatments for autism spectrum disorders: a systematic review," Annals of Clinical Psychiatry, vol. 21, no. 4, pp. 213-236, 2009.

[21] J. Whipple, "Music in intervention for children and adolescents with autism: a meta-analysis," Journal of Music Therapy, vol. 41, no. 2, pp. 90-106, 2004.

[22] C. Gold, T. Wigram, and C. Elefant, "Music therapy for autistic spectrum disorder," Cochrane Database of Systematic Reviews, no. 2, p. CD004381, 2006.

[23] S. Kingsnorth, K. Treurnicht Naylor, A. Lamont, P. McKeever, and C. MacArthur, "The effectiveness of music in pediatric healthcare: a systematic review of randomized controlled trials," Evidence-Based Complementary and Alternative Medicine, vol. 2011, Article ID 464759, 18 pages, 2011.
[24] L. A. Cullen-Powell, J. H. Barlow, and D. Cushway, "Exploring a massage intervention for parents and their children with autism: the implications for bonding and attachment," Journal of Child Health Care, vol. 9, no. 4, pp. 245-255, 2005.

[25] S. Beider and C. A. Moyer, "Randomized controlled trials of pediatric massage: a review," Evidence-Based Complementary and Alternative Medicine, vol. 4, no. 1, pp. 23-34, 2007.

[26] L. M. T. Silva, A. Cignolini, R. Warren, S. Budden, and A. Skowron-Gooch, "Improvement in sensory impairment and social interaction in young children with autism following treatment with an original qigong massage methodology," American Journal of Chinese Medicine, vol. 35, no. 3, pp. 393406, 2007.

[27] H. Allam, N. G. Eldine, and G. Helmy, "Scalp acupuncture effect on language development in children with autism: a pilot study," Journal of Alternative and Complementary Medicine, vol. 14, no. 2, pp. 109-114, 2008.

[28] D. K. L. Cheuk, V. Wong, and W. X. Chen, "Acupuncture for autism spectrum disorders (ASD)," Cochrane Database of Systematic Reviews, vol. 9, Article ID CD007849, 2011.

[29] S.L. Hyman and S. E. Levy, "Dietary, complementary and alternative therapies," in Evidence-Based Practices and Treatments for Children with Autism, B. Reichow, P. Doehring, D. V. Cicchetti, and F. R. Volkmar, Eds., 2011.

[30] A. S. Chan, Dejian Mind-Body Intervention: Clinical Application of Shaolin Chanwuyi, Chanwuyi Publishing, Hong Kong, 4th edition, 2009.

[31] A. S. Chan, M. C. Cheung, W. J. Tsui, S. L. Sze, and D. Shi, "Dejian mind-body intervention on depressive mood of community-dwelling adults: a randomized controlled trial," Evidence-Based Complementary and Alternative Medicine, vol. 2011, Article ID 473961, 8 pages, 2011.

[32] A. S. Chan, S. L. Sze, M. C. Cheung, Y. M. Y. Han, W. W. M. Leung, and D. Shi, "Dejian mind-body intervention improves the cognitive functions of a child with autism," Evidence-based Complementary and Alternative Medicine, vol. 2011, Article ID 549254, 7 pages, 2011.

[33] A. S. Chan, S. L. Sze, and D. Shi, "Traditional Chinese mindbody exercises improve self control ability of an adolescent with Asperger's disorder," Journal of Psychology in Chinese Societies, vol. 9, pp. 225-239, 2008.

[34] A. S. Chan, S. L. Sze, M. C. Cheung, J. M. K. Lam, and D. Shi, "Dejian mind-body intervention improves the functioning of a patient with chronic epilepsy: a case report," Cases Journal, vol. 2, Article ID 9080, 7 pages, 2009.

[35] A. S. Chan, Q. Y. Wong, S. L. Sze, P. P. K. Kwong, Y. M. Y. Han, and M. C. Cheung, "A Chinese Chan-based mind-body intervention improves sleep on patients with depression: a randomized controlled trial," The Scientific World Journal, vol. 2012, Article ID 235206, 10 pages, 2012.

[36] A. S. Chan, Q. Y. Wong, S. L. Sze et al., "A Chinese Chan-based mind-body intervention for patients with depression," Journal of Affective Disorders. In press.

[37] R. Raymaekers, J. Van Der Meere, and H. Roeyers, "Eventrate manipulation and its effect on arousal modulation and response inhibition in adults with high functioning autism," Journal of Clinical and Experimental Neuropsychology, vol. 26, no. 1, pp. 74-82, 2004.

[38] M. Langen, A. Leemans, P. Johnston et al., "Fronto-striatal circuitry and inhibitory control in autism: findings from diffusion tensor imaging tractography," Cortex, vol. 48, no. 2, pp. 183-193, 2012. 
[39] J. Watanabe, M. Sugiura, K. Sato et al., "The human prefrontal and parietal association cortices are involved in no-go performances: an event-related fmri study," Neuroimage, vol. 17, no. 3, pp. 1207-1216, 2002.

[40] R. Elliott and R. J. Dolan, "Activation of different anterior cingulate foci in association with hypothesis testing and response selection," Neuroimage, vol. 8, no. 1, pp. 17-29, 1998.

[41] M. M. Botvinick, J. D. Cohen, and C. S. Carter, "Conflict monitoring and anterior cingulate cortex: an update," Trends in Cognitive Sciences, vol. 8, no. 12, pp. 539-546, 2004.

[42] S. F. Taylor, E. R. Stern, and W. J. Gehring, "Neural systems for error monitoring: recent findings and theoretical perspectives," Neuroscientist, vol. 13, no. 2, pp. 160-172, 2007.

[43] A. S. Chan, Y. M. Y. Han, W. W. M. Leung, C. Leung, V. C. N. Wong, and M. C. Cheung, "Abnormalities in the anterior cingulate cortex associated with attentional and inhibitory control deficits: a neurophysiological study on children with autism spectrum disorders," Research in Autism Spectrum Disorders, vol. 5, no. 1, pp. 254-266, 2011.

[44] E. A. Hazlett, M. S. Buchsbaum, P. Hsieh et al., "Regional glucose metabolism within cortical brodmann areas in healthy individuals and autistic patients," Neuropsychobiology, vol. 49, no. 3, pp. 115-125, 2004.

[45] M. M. Haznedar, M. S. Buchsbaum, E. A. Hazlett, E. M. LiCalzi, C. Cartwright, and E. Hollander, "Volumetric analysis and three-dimensional glucose metabolic mapping of the striatum and thalamus in patients with autism spectrum disorders," American Journal of Psychiatry, vol. 163, no. 7, pp. 1252-1263, 2006.

[46] Y. Agam, R. M. Joseph, J. J. S. Barton, and D. S. Manoach, "Reduced cognitive control of response inhibition by the anterior cingulate cortex in autism spectrum disorders," Neuroimage, vol. 52, no. 1, pp. 336-347, 2010.

[47] American Psychiatric Association, Diagnostic and Statistical Manual of Mental Disorders, Washington, DC, USA, 4th edition, 2002.

[48] C. Lord, M. Rutter, and A. L. Couteur, "Autism diagnostic interview-revised: a revised version of a diagnostic interview for caregivers of individuals with possible pervasive developmental disorders," Journal of Autism and Developmental Disorders, vol. 24, no. 5, pp. 659-685, 1994.

[49] A. S. Kaufman, J. C. Kaufman, R. Balgopal, and J. E. McLean, "Comparison of three WISC-III short forms: weighing psychometric, clinical, and practical factors," Journal of Clinical Child Psychology, vol. 25, no. 1, pp. 97-105, 1996.

[50] R. L. Thorndike, E. P. Hagen, and J. M. Sattler, Stanford-Binet Intelligence Test, Riverside, Ill, USA, 1986, 4th edition.

[51] G. H. Klem, H. O. Lüders, H. H. Jasper, and C. Elger, "The ten-twenty electrode system of the international federation. the international federation of clinical neurophysiology," Electroencephalography and Clinical Neurophysiology, vol. 52, pp. 371-375, 1999.

[52] R. Brickenkamp, Aufmerksamkeits-Belastungs-Test, Test d2, Hogrefe, Gottingen, Germany, 1981.

[53] J. Williams, V. Rickert, J. Hogan et al., "Children's color trails," Archives of Clinical Neuropsychology, vol. 10, no. 3, pp. 211223, 1995.

[54] M. Regard, E. Strauss, and P. Knapp, "Children's production on verbal and non-verbal fluency tasks," Perceptual and Motor Skills, vol. 55, no. 3, pp. 839-844, 1982.

[55] S. N. Mattson, A. M. Goodman, C. Caine, D. C. Delis, and E. P. Riley, "Executive functioning in children with heavy prenatal alcohol exposure," Alcoholism: Clinical and Experimental Research, vol. 23, no. 11, pp. 1808-1815, 1999.
[56] Autism Treatment Evaluation Checklist, "Reliabilities and score distributions," 2000, http://www.autism.com/ari/atec/ atec_report.htm.

[57] H. Asada, Y. Fukuda, S. Tsunoda, M. Yamaguchi, and M. Tonoike, "Frontal midline theta rhythms reflect alternative activation of prefrontal cortex and anterior cingulate cortex in humans," Neuroscience Letters, vol. 274, no. 1, pp. 29-32, 1999.

[58] R. Ishii, K. Shinosaki, S. Ukai et al., "Medial prefrontal cortex generates frontal midline theta rhythm," Neuroreport, vol. 10, no. 4, pp. 675-679, 1999.

[59] D. Pizzagalli, R. D. Pascual-Marqui, J. B. Nitschke et al., "Anterior cingulate activity as a predictor of degree of treatment response in major depression: evidence from brain electrical tomography analysis," American Journal of Psychiatry, vol. 158, no. 3, pp. 405-415, 2001.

[60] R. D. Pascual-Marqui, D. Lehmann, T. Koenig et al., "Low resolution brain electromagnetic tomography (loreta) functional imaging in acute, neuroleptic-naive, first-episode, productive schizophrenia," Psychiatry Research: Neuroimaging, vol. 90, no. 3, pp. 169-179, 1999.

[61] R. D. Pascual-Marqui, C. M. Michel, and D. Lehmann, "Low resolution electromagnetic tomography: a new method for localizing electrical activity in the brain," International Journal of Psychophysiology, vol. 18, no. 1, pp. 49-65, 1994.

[62] W. J. Craig, "Health effects of vegan diets," American Journal of Clinical Nutrition, vol. 89, no. 5, 2009.

[63] G. E. Fraser, "Vegetarian diets: what do we know of their effects on common chronic diseases?" American Journal of Clinical Nutrition, vol. 89, no. 5, pp. 1607S-1612S, 2009.

[64] B. L. Beezhold, C. S. Johnston, and D. R. Daigle, "Vegetarian diets are associated with healthy mood states: a cross-sectional study in seventh day adventist adults," Nutrition Journal, vol. 9, article 26, 2010.

[65] E. B. Isaacs, D. G. Gadian, S. Sabatini et al., "The effect of early human diet on caudate volumes and IQ," Pediatric Research, vol. 63, no. 3, pp. 308-314, 2008.

[66] C. R. Gale, C. N. Martyn, L. D. Marriott et al., "Dietary patterns in infancy and cognitive and neuropsychological function in childhood," Journal of Child Psychology and Psychiatry and Allied Disciplines, vol. 50, no. 7, pp. 816-823, 2009.

[67] D. Benton, "The influence of dietary status on the cognitive performance of children," Molecular Nutrition and Food Research, vol. 54, no. 4, pp. 457-470, 2010. 


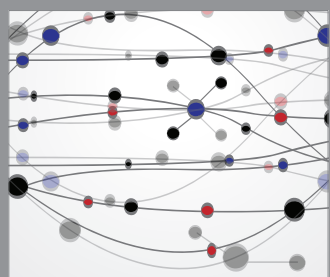

The Scientific World Journal
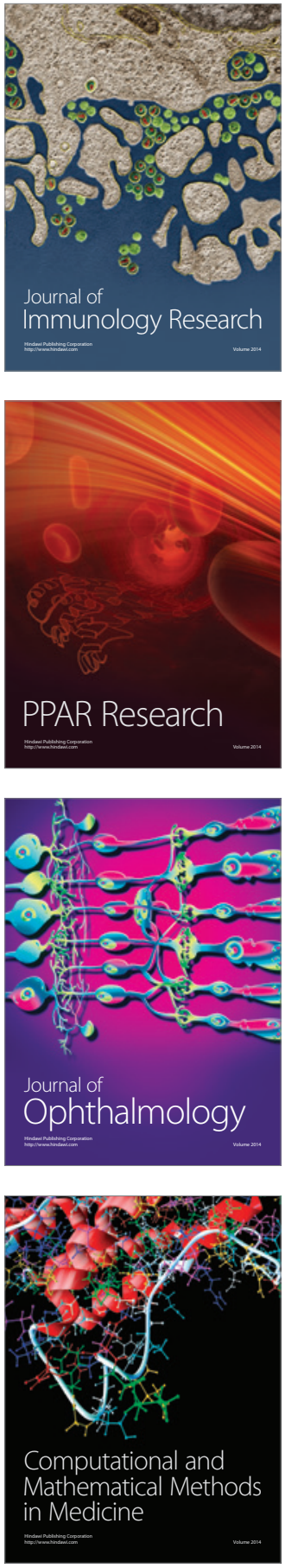

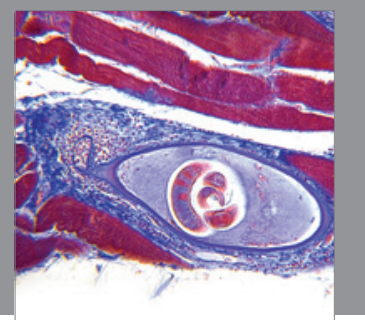

Gastroenterology

Research and Practice
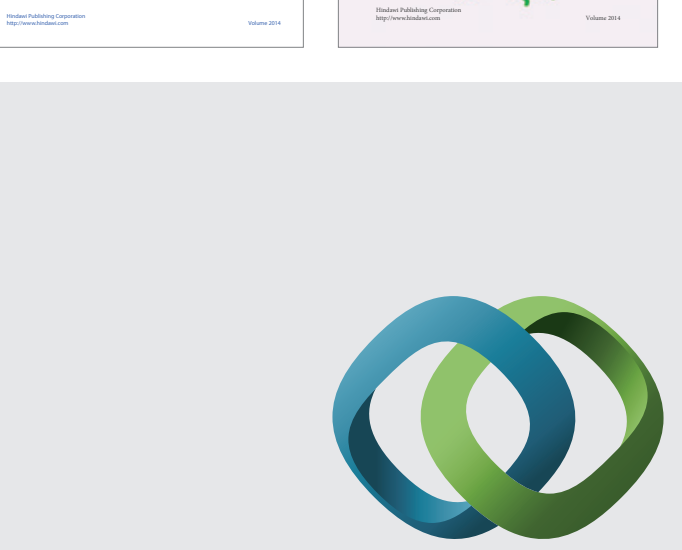

\section{Hindawi}

Submit your manuscripts at

http://www.hindawi.com
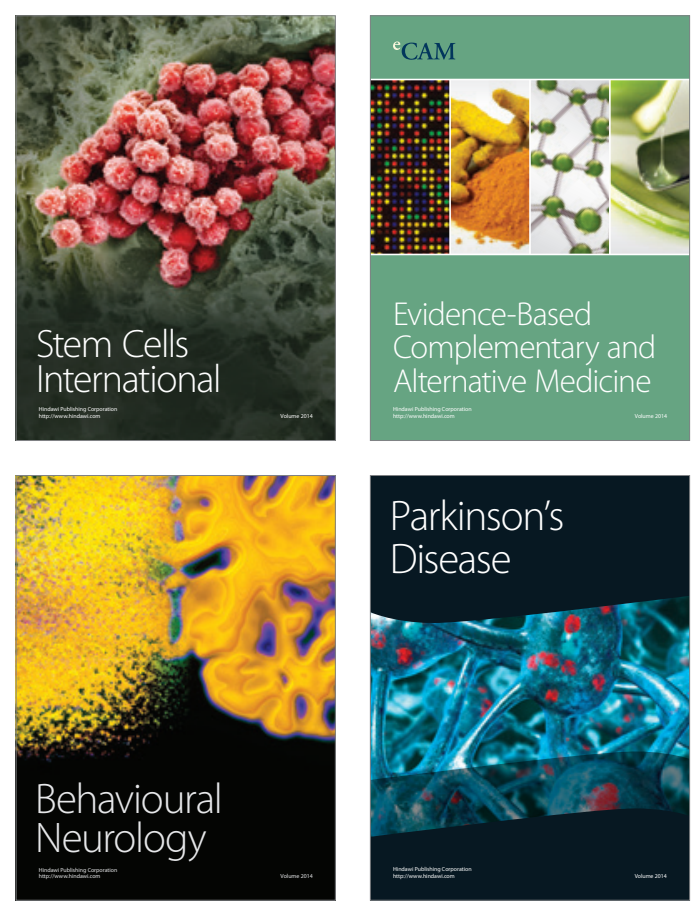

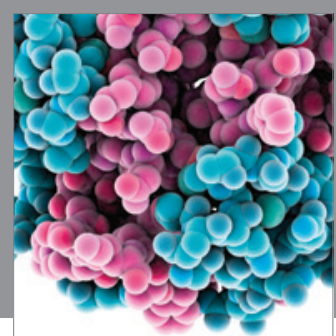

Journal of
Diabetes Research

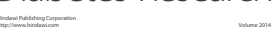

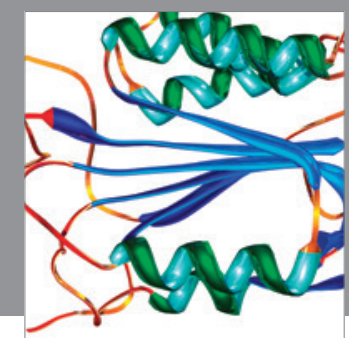

Disease Markers
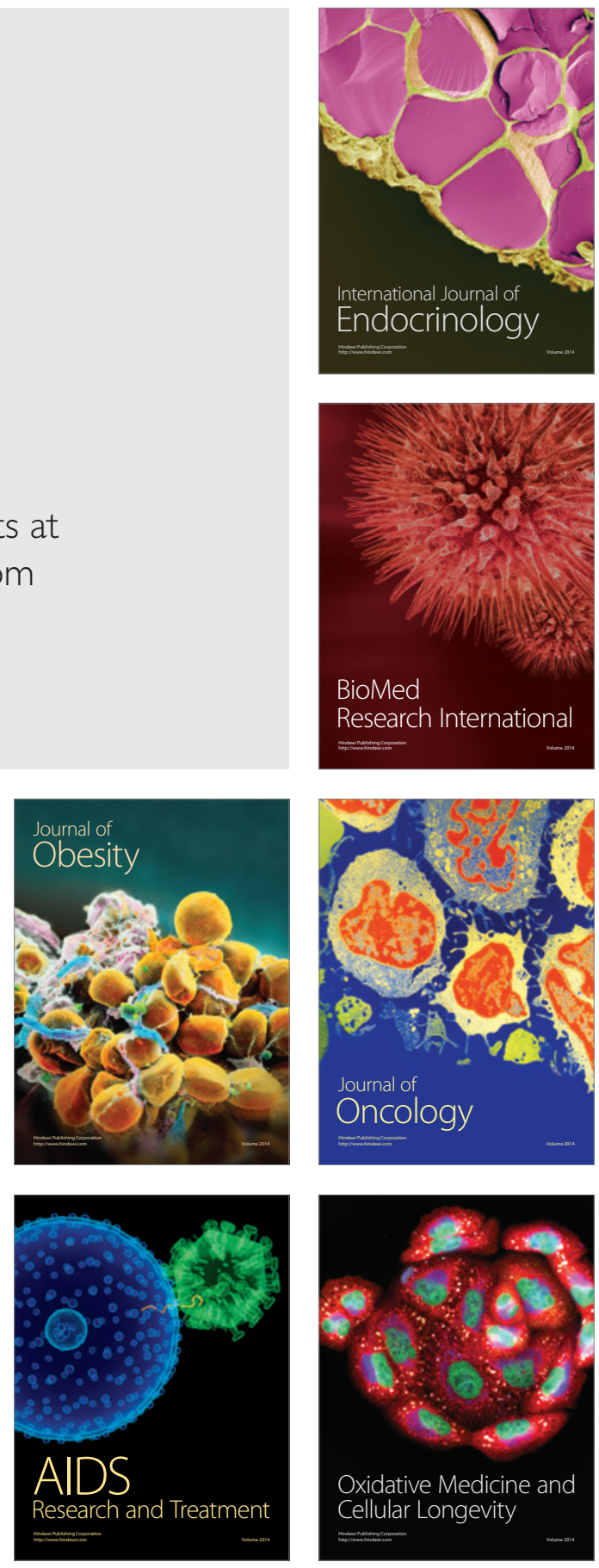